\title{
A Mandibular Advancement Device Attenuates the Abnormal Morphology and Function of Mitochondria from the Genioglossus in OSAHS Rabbits
}

Chunyan Liu

Hebei Medical University

Shilong Zhang

Hebei Medical University

Dechao Zhu

Hebei Medical University https://orcid.org/0000-0002-8237-1731

Dengying Fan

Hebei Medical University

Yahui Zhu

Hebei Medical University

Wenjing Kang

Hebei Medical University

Haiyan Lu ( $D$ luhaiyan67@163.com )

Hebei Medical University

Jie Wang

Hebei Medical University

\section{Research}

Keywords: Obstructive sleep apnea-hypopnea syndrome, Mandibular advancement device, Genioglossus, Mitochondria

Posted Date: August 5th, 2021

DOI: https://doi.org/10.21203/rs.3.rs-622336/v1

License: (9) (1) This work is licensed under a Creative Commons Attribution 4.0 International License. Read Full License 


\section{Abstract}

Background: To examine the morphology and function of mitochondria from the genioglossus in a rabbit model of obstructive sleep apnea-hypopnea syndrome (OSAHS), as well as these factors after insertion of a mandibular advancement device (MAD).

Methods: Thirty male New Zealand white rabbits were randomized into three groups: control, OSAHS and MAD, with 10 rabbits in each group. Animals in Group OSAHS and Group MAD were induced to develop OSAHS by injection of gel into the submucosal muscular layer of the soft palate. The rabbits in Group MAD were fitted with a MAD. The animals in the control group were not treated. Further, polysomnography (PSG) and CBCT scan were used to measure MAD effectiveness. CBCT of the upper airway and PSG suggested that MAD was effective. Rabbits in the three groups were induced to sleep for 4-6 hours per day for 8 consecutive weeks. The genioglossus was harvested and detected by optical microscopy and transmission electron microscopy. The mitochondrial membrane potential was determined by laser confocal microscopy and flow cytometry. Mitochondrial complex I and IV activities were detected by mitochondrial complex assay kits.

Results: OSAHS-like symptoms were induced successfully in Group OSAHS and rescued by MAD treatment. The relative values of the mitochondrial membrane potential, mitochondrial complex I activity and complex IV activity were significantly lower in Group OSAHS than in the control group; however, there was no significant difference between Group MAD and the control group. The OSAHS-induced injury and the dysfunctional mitochondria of the genioglossus muscle were reduced by MAD treatment.

Conclusion: Damaged mitochondrial structure and function were induced by OSAHS and could be attenuated by MAD treatment.

\section{Contributions To The Literature}

1. This study suggested that MAD treatment attenuated the dysfunction of genioglossus mitochondria caused by OSAHS, which could expand our understanding of the effect of MAD on the genioglossus during OSAHS treatment.

2. This study will provide further evidence for the treatment of OSAHS with appliance in clinical practice.

3. This study will enrich our knowledge on the advantages of MAD treatment for OSAHS, to further guide clinicians' treatment options for OSAHS.

\section{Background}

Obstructive sleep apnea-hypopnea syndrome (OSAHS) is a serious, potentially life-threatening condition characterized by loud snoring and recurrent episodes of partial or complete upper airway (UA) collapse occurring during sleep(1). OSAHS is the most common respiratory sleep disorder, and its prevalence is 
estimated to be up to $10 \%$ in middle-aged men and $3 \%$ in middle-aged women(2). The UA is susceptible to collapse during sleep due to its lack of rigid bony support(3). Its lumen size depends on the balance between the UA dilator tension and upper airway negative pressure. Normally, the UA dilator muscles are responsible for maintaining UA patency, especially the genioglossus, which is the most important pharyngeal dilator muscle and plays a key role in the maintenance of UA patency during sleep(4).

Treatment options for OSAHS include behavioral modification, continuous positive airway pressure (CPAP), mandibular advancement device (MAD)(5), surgical procedures(6), electrical stimulation(7) and pharmacological treatments. CPAP is recommended as the first-line therapy according to the treatment guidelines for patients with moderate-to-severe OSAHS(8). CPAP can improve snoring, the apnea/hypopnea index $(\mathrm{AHI})$ and daytime sleepiness through an air splint to maintain UA patency $(9)$. However, CPAP is not accepted by all patients; thus, the effectiveness is unsatisfactory in some patients due to the limited adherence to this treatment(10). MAD has the advantages of being noninvasive, low cost, comfortable and easy to carry. Therefore, this option is very popular for most OSAHS patients, especially for those who are neither able nor willing to tolerate CPAP therapy or surgery. To date, scholars pay more attention on changes in subjective symptoms, sleep quality and airway structure during MAD therapy for OSAHS. Our previous studies(11) found that abnormal contractility and fiber type distribution of the genioglossus could be caused by OSAHS. However, whether the mitochondria participate in these tissue changes is unclear. As skeletal muscle, the function of genioglossus is closely related to fiber types and energy metabolism(11). Thus, OSAHS-induced abnormality of genioglossus function may correlate with mitochondrial dysfunction. Therefore, the aim of this study was to investigate whether OSAHS induces mitochondrial structural damage and dysfunction. Moreover, considering the effectiveness of MAD in treating patients with mild-to-moderate OSAHS, we investigated whether MAD treatment could be effective against OSAHS-induced mitochondrial structural damage and dysfunction.

\section{Methods}

All experimental protocols and animal studies were submitted to and approved by local animal committee. The flowchart of the study was shown in Fig. 1. All methods in this study were performed in accordance with the medical ethics committee, and an additional file shows this in more details [see Additional file 1]. Animal use and care was in accordance with the guidelines of the medical ethics committee for the housing and care of animals bred, supplied and used for scientific purposes. All experiments were performed in accordance with relevant guidelines and regulations. The study was carried out in compliance with the ARRIVE guidelines.

\section{Animal Model Development}

Thirty New Zealand white rabbits (initial weight, $3 \mathrm{~kg}-3.5 \mathrm{~kg}$ ) were equally divided into three experimental groups, namely the control group, Group OSAHS and Group MAD, with 10 animals in each group. The animals were kept at $22^{\circ} \mathrm{C}-25^{\circ} \mathrm{C}$ with free access to food and water. The animal models were developed as described in our previous studies $(11,13,14)$. Briefly, OSAHS was induced by Medical Sodium 
Hyaluronate Gel (Shanghai Qisheng Biological Preparation Co., Ltd. Shanghai, China) injection via the submucosal muscular layer at the center of the soft palate, approximately $1.5 \mathrm{~cm}$ away from the junction of the hard and soft palates. The animals in Group MAD were given MAD to alleviate the OSAHS symptoms. Animals in the control group were not given any treatment.

\section{Confirmation of induced OSAHS}

Cone-beamcomputedtomography (CBCT) and polysomnography (PSG) were conducted. A CBCT scan of the UA was performed with a CBCT machine (KaVo 3D eXam, USA). The parameters were set as follows: the current size of $5 \mathrm{~mA}$, the voltage magnitude of $120 \mathrm{kV}$, the scan time of $17.8 \mathrm{~s}$ and the layer thickness of $0.3 \mathrm{~mm}$. Then, 3D reconstruction by the manufacturer was conducted, and the retropalatal space in the UA was examined from the top, $1 / 4,1 / 2$, and $3 / 4$ levels to the bottom of the soft palate(15).

PSG (Rembrandt Embla Polysomnography System, Reykjavik, Iceland) was used to monitor the sleep parameters. PSG recordings were conducted as described in our previous studies $(11,13)$. Briefly, the rabbits were equipped with surface electrodes taped on the skull, face, and chest for monitoring the electroencephalogram, electrooculogram and respiration. Nasal air flow, respiratory movements, blood oxygen saturation ( $\mathrm{SaO} 2)$, and $\mathrm{AHI}$ were scored for all animals, the scoring method was the same as that we previously used $(11,13,15)$.

OSAHS rabbits in Group MAD were given the MAD. This device was made of self-curing composite resin and bonded to the upper incisors with glass ionomer, with a $30^{\circ}$ inclined plane to the long axis of the upper incisors $(11,13)$. The mandible was guided forward by 3-4 mm. After 3-5 days of adaptation, no rabbits had difficulty eating or had signs of distress.

No animals showed difficulties eating or drinking. Chloral hydrate was infused orally to induce sleep in a supine position for 4-6 hours per day for the next eight weeks. Basic health data, including body weight and behavior, were recorded at 2 weeks and 8 weeks.

\section{Preparation of the genioglossus muscle tissues}

The observation period lasted for 8 weeks. Finally, the genioglossus was harvested and fixed in $10 \%$ buffered formalin for 24 hours and then routinely embedded in paraffin. The sections were stained with hematoxylin/eosin and examined under light microscopy (Olympus, Japan). A portion of the genioglossus was quickly dissected into a $1 \mathrm{~mm} \times 1 \mathrm{~mm} \times 3 \mathrm{~mm} 3$ piece, which was fixed in a $4 \%$ cryopreservation glutaraldehyde solution and then underwent preparation for conventional transmission electron microscopy to examine the ultrastructure.

\section{Mitochondrial functions of the genioglossus}

Mitochondrial isolation of the genioglossus was performed in strict accordance with the instructions for the mitochondria extraction kit using a buffer containing $180 \mathrm{mM} \mathrm{KCl}, 10 \mathrm{mM}$ EDTA(Ethylene Diamine Tetraacetic Acid), and $0.5 \%$ albumin at $\mathrm{pH} 7.4$. The mitochondrial membrane potential $(\Delta \Psi \mathrm{m})$ was estimated with a membrane electrical potential assay kit. After JC-1 ( CBIC2) fluorescent probe loading, 
flow cytometric analysis was conducted to quantitatively determine the relative value of the mitochondrial membrane potential. The fluorescence signals of the JC-1 monomer (red fluorescence) and polymer (green fluorescence) were obtained in the fluorescence 1 (FL1), and fluorescence 2 (FL2) detectors. A flow diagram was obtained from flow cytometry and analyzed by Exp032ADC analysis software. Comparisons of the fluorescence intensities of red fluorescence and green fluorescence could reflect the mitochondrial membrane potential. The mitochondrial membrane potential was qualitatively determined by laser confocal microscopy. The excitation wavelengths were $488 \mathrm{~nm}$ and $525 \mathrm{~nm}$. The appearance of green fluorescence or a decreased intensity of red fluorescence indicated that the mitochondrial membrane potential decreased.

\section{Mitochondrial complex I and IV activities}

The quantitative detection was in strict accordance with the mitochondrial respiratory chain complex I activity and complex IV activity assay kit. The activities of the samples were calculated according to the formula shown in the kit.

The results were analyzed with SPSS 22.0 software (SPSS, Chicago, USA). Different parameters were assessed for normally distributed data. All data are expressed as the mean \pm SD. The statistical significance of differences was assessed by analysis of variance (ANOVA) after normality and variance equality were tested, and the LSD test was used to compare the differences among three groups. A p value $<0.05$ was considered statistically significant.

\section{Results}

\section{Rabbit behaviors}

At baseline, 2 weeks and 8 weeks after modeling, there was no significant difference in body weight and food intake among the three groups (Fig. 2).

\section{The induced OSAHS-like symptoms}

OSAHS-like symptoms were induced successfully in Group OSAHS, which showed snoring and apnea or hypopnea during supine sleep, accompanied by interrupted sleep, but these symptoms were not detected in the animals in Group MAD and the controls.

The retropalatal space in the UA was significantly decreased in Group OSAHS $(p<0.05)$, and MAD enlarged the retropalatal UA (Fig. 3). PSG also showed significantly higher AHI and lower SaO2 levels in Group OSAHS than in Group MAD and the control group ( $p<0.05)$ (Fig. 4). All of these results suggested that OSAHS was successfully induced and that MAD effectively alleviated the OSAHS-like symptoms.

\section{The morphology of the genioglossus}


HE staining showed that varying degrees of disordered arrangement of the muscle fibers in Group OSAHS. Disorders and degeneration of the genioglossus fibers were not detected in Group MAD and in the control group (Fig. 5).

As for the ultrastructure of the genioglossus, the control group showed regular myofibrils. In Group OSAHS, discontinuous myofibrils, swelling and degeneration of the mitochondria, dilation of the mitochondria and disruption of the cristae were detected. Some mitochondria dissolved or even disappeared. Compared with Group OSAHS, the ultrastructural changes of genioglossus in Group MAD were less severe, with a mildly disordered arrangement of the myofibrils and mild mitochondrial degeneration and edema (Fig. 5).

\section{Mitochondrial functions of the genioglossus}

Flow cytometric analysis and laser scanning confocal microscopy showed that the relative value of the mitochondrial membrane potential was significantly lower in Group OSAHS than that in the control group. There was no significant difference in the relative value of the mitochondrial membrane potential between Group MAD and the control group (Fig. 6).

Mitochondrial complex I activity and complex IV activity were significantly decreased in Group OSAHS compared with the control group ( $p<0.05)$. However, MAD treatment attenuated the effect of OSAHS on complex I activity and complex IV activities (Fig. 7).

\section{Discussion}

In this study, we detected genioglossus injury, dysfunctional mitochondria and decreased mitochondrial respiratory chain complex I and

IV activities of the genioglossus in a rabbit model of OSAHS, these results described a possible mechanism, supporting of our previous reports on genioglossus fatigue. As in other diseases, research based on animal models is crucially important in OSAHS $(11,13)$. The model of intermittent hypoxia was a widely documented method of inducing OSAHS(17). Although this model appeared to demonstrate similar symptoms to OSAHS patients, it was obviously limited by the absence of recurrent UA obstructions, apnea, increased inspiratory effort and sleep fragmentation(18). In these cases, the aim was to obtain an OSAHS model induced by UA obstruction and demonstrate all the typical characteristics of OSAHS, such as intermittent hypoxia, increased inspiratory effort and sleep fragmentation. An approach to induce OSAHS and insert MAD in rabbits was developed in our laboratory. Technical success was achieved, and the MAD was well tolerated in the rabbits, as shown by the body weight and food intake.

This study found that OSAHS caused abnormal morphology of genioglossus, such as degeneration of the genioglossus fibers and disordered mitochondrial ultrastructure, including discontinuous myofibrils, dilation of the mitochondria and disruption of the cristae. Accordingly, we previously found that OSAHS resulted in genioglossus fatigue in vitro(11). However, the detailed molecular mechanisms remain to be 
elucidated. Previous reports demonstrated that the genioglossus may be more vulnerable to fatigue in OSAHS patients $(19,20)$ and animal models of OSAHS than in controls $(21-23)$. Therefore, identification of an underlying contributory mechanism of genioglossus fatigue is important and has therapeutic implications. The function of skeletal muscle is intimately linked to the proper function of mitochondria because mitochondria constitute the main energy supply for contraction of skeletal muscle. We intended to examine whether genioglossus fatigue was related to mitochondria. Consistent with our hypothesis, mitochondrial abnormalities, such as decreased mitochondrial membrane potential and decreased mitochondrial respiratory chain complex activity, as well as dysfunctional mitochondrial ultrastructure of genioglossus, were revealed in Group OSAHS. These findings could explain why the abnormal changes in the structure and contractile properties of the genioglossus were observed in our previous studies.

With regard to $\Delta \Psi \mathrm{m}$, our data showed that the mitochondria isolated from the animals with OSAHS had a lower $\Delta \Psi \mathrm{m}$ than those of the controls. Since mitochondrial membrane potential is a key indicator reflecting mitochondrial function and $\Delta \Psi \mathrm{m}$ provides reliable information on muscle function and dysfunction(24), the data suggested that OSAHS could indeed cause mitochondrial dysfunction. However, a simple analysis of mitochondrial membrane potential is insufficient to determine the mechanisms underlying the damage to genioglossus function and the effectiveness of the MAD treatment. Since respiratory chain complexes I, III and IV generate $\Delta \Psi \mathrm{m}$ as a result of energy transfer through the electron transport chain(25), to further clarify the genioglossus mitochondrial function, we evaluated the mitochondrial respiratory chain complexes in the present study. We found that these complexes were also severely affected by OSAHS; thus, we confirmed that OSAHS clearly affects genioglossus mitochondrial function.

To the best of our knowledge, this is the first study examining genioglossus mitochondrial functionality in OSAHS models and after MAD treatment. We hypothesize that the mitochondrial dysfunction and morphological abnormalities observed in the animals with OSAHS are due to one or more of the following causes. First, chronic intermittent hypoxia during repeated apnea or hypopnea results in mitochondrial dysfunction. UA closure during sleep is associated with oxygen desaturation, which terminates when an arousal transiently interrupts sleep. Then, apneas can recur as sleep resumes, contributing to the pathogenesis of chronic intermittent hypoxia. A study showed that hypoxia in OSAHS patients impaired UA muscle activity(26). Another report demonstrated that hypoxia could increase oxidative stress and impair mitochondrial function in mouse skeletal muscle because hypoxia affected both the mitochondrial phosphorylation efficiency and the coupling between respiration and ATP synthesis. Similarly, a previous study showed that the hypoxia-induced mitochondrial dysfunction and the inner and outer mitochondrial membrane integrity were significantly affected by hypoxia exposure(28). Therefore, mitochondrial dysfunction may be closely related to chronic intermittent hypoxia, the most basic physical characteristic of OSAHS. Second, repeated bursts of forceful contraction may lead to mitochondrial abnormalities in genioglossus. Genioglossus actively compensate for the narrowed upper airway in OSAHS during wakefulness, which is supported by the research that OSAHS patients have increased GG activation relative to controls during wakefulness(29) and there was greater reduction in GG activation in OSA patients than in controls after CPAP treatment, implying that the enhanced activity is a compensatory 
response(30). Therefore, repeated forceful contraction of the genioglossus may lead to mitochondrial abnormalities. Dysfunction of UA dilator muscles is closely involved in the pathophysiology of OSAHS(1). In OSAHS patients, the genioglossus has been shown to be structurally and functionally abnormal, with elevated levels of activation while awake(31). The genioglossus, when activated, protracts the tongue and results in increased airway patency and further prevents collapse and subsequent apneic events(32). When performing repeated tongue protrusions, the genioglossus exerts repeated bursts of forceful contraction at the end of each obstructive apnea; thus, traumatic muscle contractions have a negative effect on mitochondrial structure and function following repeated activation during the night. Our results suggested that genioglossus injury, including histopathological muscle changes and metabolic disturbances, may be the result of OSAHS. The genioglossus in Group OSAHS was characterized by morphological abnormalities, together with decreased abnormal mitochondrial functions of the genioglossus, leaving the UA susceptible to collapse and leading to a vicious cycle of increasingly severe episodes of obstruction during sleep.

Our previous work has shown that genioglossus fatigue related to OSAHS could be corrected by MAD treatment(11), suggesting that genioglossus fatigue was related to OSAHS, and MAD may protect against injury. In the present study, the insertion of the MAD in rabbits with OSAHS significantly improved the genioglossus mitochondrial morphology and function, which were similar to those in the normal controls. The precise mechanisms of MAD therapy are still unclear. However, MAD directly increased the size of the pharyngeal airway(33). The genioglossus functions as a dilator of the pharyngeal airway and is responsible for maintaining patency of the UA(31). The genioglossus was reported to generate the main protrusive force of the tongue, and its contraction and relaxation substantially affected the dimensions of the UA(34). The potential mechanics that may account for the improvements in genioglossus following MAD insertion could be through the augmentation of the pharyngeal airway and the activation of the genioglossus. The delivery of MAD to the rabbits with OSAHS could be associated with resting of the genioglossus. Furthermore, chronic intermittent hypoxia was eliminated following the insertion of MAD. It was reported that there was no difference in the level of GG activation between OSA patients and healthy individuals when on fully therapeutic CPAP(35). A recent study identified significant increases in genioglossus activity following placement of the $\operatorname{MAD}(36)$. This finding suggests that the anti-apnea effects and the increased activity represent two of the most important mechanisms by which MAD protects the genioglossus against OSAHS-induced injury. Ultrastructural observation by transmission electron microscopy indicated that MAD treatment could attenuate the mitochondrial swelling and disrupted cristae in the genioglossus induced by OSAHS.

\section{Conclusion}

In summary, our results suggested that OSAHS caused damage to the muscle mitochondrial morphology and function. MAD treatment attenuated the deleterious effects of OSAHS on the genioglossus mitochondria. This protective effect was mediated by the MAD-mediated enlargement of the UA. 


\section{Abbreviations}

OSAHS: Obstructive sleep apnea-hypopnea syndrome

MAD: Mandibular advancement device

PSG: Polysomnography

UA: Upper airway

CPAP: Continuous positive airway pressure

CBCT: Cone-beamcomputedtomography

Sa02: Blood oxygen saturation

FL1: Fluorescence 1

FL2: Fluorescence 2

\section{Declarations}

\section{Acknowledgements}

We gratefully acknowledge study participants who took their valuable time to participate in this study.

\section{Funding}

Funding/Support: This study was funded by Natural Science Foundation of China (NSFC) (Grant No.81701010), the Training Program of Clinical Medicine Talents Funded by the Government of Hebei Province (Grant No. 361029) , Youth Foundation of Hebei Education Department (Grant No. QN2017109) and Natural Science Foundation of Hebei Province(Grant No. H2021206431)

\section{Availability of data and materials}

The data underlying this article will be shared on reasonable request to the corresponding author.

\section{Authors' information}

\section{Affiliations}

Chunyan Liu, Shilong Zhang, Dechao Zhu, Dengying Fan, Yahui Zhu, Wenjing Kang, Haiyan Lu

Department of Orthodontics, School and Hospital of Stomatology, Hebei Medical University \& Hebei Key Laboratory of Stomatology \& Hebei Clinical Research Center for Oral Diseases, Shijiazhuang, 050017, PR China. 
Jie Wang

Department of Oral Pathology, School and Hospital of Stomatology, Hebei Medical University \& Hebei Key Laboratory of Stomatology \& Hebei Clinical Research Center for Oral Diseases, Shijiazhuang, 050017, PR China.

\section{Authors' Contributions}

Chunyan Liu, Haiyan Lu and Jie Wang led the conception and design of the study, was closely involved in data analysis and interpretation. Chunyan Liu, Shilong Zhang and Dechao Zhu were responsible for raising animals. Chunyan Liu, Shilong Zhang, Dechao Zhu, Dengying Fan, Yahui Zhu, Wenjing Kang were responsible for establishing animal models. Dechao Zhu, Dengying Fan, Yahui Zhu, Wenjing Kang were responsible for all the experiments and acquiring data. All authors reviewed the manuscript.

\section{Corresponding author}

Correspondence to Haiyan Lu and Jie Wang

\section{Ethics declarations}

\section{Ethics approval and consent to participate}

Approval to conduct the study was gained from the medical ethics committee in Hospital of Stomatology, Hebei Medical University.

\section{Consent for publication}

Yes

\section{Competing interests}

The authors declare that they have no competing interests.

\section{References}

1. Patil SP, Schneider H, Schwartz AR, Smith PL. Adult obstructive sleep apnea: pathophysiology and diagnosis. CHEST. [Journal Article; Research Support, N.I.H., Extramural; Review]. 2007 2007-0701;132(1):325-37.

2. Peppard PE, Young T, Barnet JH, Palta M, Hagen EW, Hla KM. Increased prevalence of sleepdisordered breathing in adults. AM J EPIDEMIOL. [Journal Article; Research Support, N.I.H., Extramural]. 2013 2013-05-01;177(9):1006-14.

3. Horner RL. The neuropharmacology of upper airway motor control in the awake and asleep states: implications for obstructive sleep apnoea. Respir Res. [Journal Article; Research Support, Non-U.S. Gov't; Review]. 2001 2001-01-20;2(5):286-94. 
4. Remmers JE, DeGroot WJ, Sauerland EK, Anch AM. Pathogenesis of upper airway occlusion during sleep. J Appl Physiol Respir Environ Exerc Physiol. [Journal Article; Research Support, U.S. Gov't, P.H.S.]. 1978 1978-06-01;44(6):931-8.

5. John CR, Gandhi S, Sakharia AR, James TT. Maxillomandibular advancement is a successful treatment for obstructive sleep apnoea: a systematic review and meta-analysis. Int J Oral Maxillofac Surg. [Journal Article; Meta-Analysis; Systematic Review]. 2018 2018-12-01;47(12):1561-71.

6. Epstein LJ, Kristo D, Strollo PJ, Friedman N, Malhotra A, Patil SP, et al. Clinical guideline for the evaluation, management and long-term care of obstructive sleep apnea in adults. J CLIN SLEEP MED. [Journal Article; Practice Guideline]. 2009 2009-06-15;5(3):263-76.

7. Certal VF, Zaghi S, Riaz M, Vieira AS, Pinheiro CT, Kushida C, et al. Hypoglossal nerve stimulation in the treatment of obstructive sleep apnea: A systematic review and meta-analysis. LARYNGOSCOPE. [Journal Article; Meta-Analysis; Review; Systematic Review]. 2015 2015-05-01;125(5):1254-64.

8. Qaseem A, Holty JE, Owens DK, Dallas P, Starkey M, Shekelle P. Management of obstructive sleep apnea in adults: A clinical practice guideline from the American College of Physicians. ANN INTERN MED. [Journal Article; Practice Guideline]. 2013 2013-10-01;159(7):471-83.

9. McDaid C, Duree KH, Griffin SC, Weatherly HL, Stradling JR, Davies RJ, et al. A systematic review of continuous positive airway pressure for obstructive sleep apnoea-hypopnoea syndrome. SLEEP MED REV. [Comparative Study; Journal Article; Research Support, Non-U.S. Gov't; Review; Systematic Review]. 2009 2009-12-01;13(6):427-36.

10. Hirshkowitz M, Sharafkhaneh A. Positive airway pressure therapy of OSA. Semin Respir Crit Care Med. [Journal Article; Review]. 2005 2005-02-01;26(1):68-79.

11. Liu CY, Lu HY, Dong FS, Ma WS, Wang J, Hu XY, et al. Effects of a mandibular advancement device on genioglossus in obstructive sleep apnoea hypopnea syndrome. Eur J Orthod. [Comparative Study; Journal Article]. 2015 2015-06-01;37(3):290-6.

12. Pathi B, Kinsey ST, Locke BR. Influence of reaction and diffusion on spatial organization of mitochondria and effectiveness factors in skeletal muscle cell design. BIOTECHNOL BIOENG. [Journal Article; Research Support, U.S. Gov't, Non-P.H.S.]. 2011 2011-08-01;108(8):1912-24.

13. Lu HY, Dong F, Liu CY, Wang J, Liu Y, Xiao W. An animal model of obstructive sleep apnoea-hypopnea syndrome corrected by mandibular advancement device. Eur J Orthod. [Journal Article]. 2015 201506-01;37(3):284-9.

14. Liu C, Kang W, Zhang S, Qiao X, Yang X, Zhou Z, et al. Mandibular Advancement Devices Prevent the Adverse Cardiac Effects of Obstructive Sleep Apnea-Hypopnea Syndrome (OSAHS). Sci Rep. [Journal Article; Research Support, Non-U.S. Gov't]. 2020 2020-02-25;10(1):3394.

15. Zhu D, Kang W, Zhang S, Qiao X, Liu J, Liu C, et al. Effect of mandibular advancement device treatment on HIF-1alpha, EPO and VEGF in the myocardium of obstructive sleep apnea-hypopnea syndrome rabbits. Sci Rep. [Journal Article; Research Support, Non-U.S. Gov't]. 2020 2020-0806;10(1):13261. 
16. Kemnitz JW, Schultz-Darken N, Tapscott SJ. An IACUC perspective on animal models of sleepdisordered breathing. ILAR J. [Journal Article]. 2009 2009-01-20;50(3):312-3.

17. Farre R, Montserrat JM, Navajas D. Morbidity due to obstructive sleep apnea: insights from animal models. CURR OPIN PULM MED. [Journal Article; Research Support, Non-U.S. Gov't; Review]. 2008 2008-11-01;14(6):530-6.

18. Nacher M, Farre R, Montserrat JM, Torres M, Navajas D, Bulbena O, et al. Biological consequences of oxygen desaturation and respiratory effort in an acute animal model of obstructive sleep apnea (OSA). SLEEP MED. [Journal Article; Research Support, Non-U.S. Gov't]. 2009 2009-09-01;10(8):892-7.

19. Eckert DJ, Lo YL, Saboisky JP, Jordan AS, White DP, Malhotra A. Sensorimotor function of the upperairway muscles and respiratory sensory processing in untreated obstructive sleep apnea. J Appl Physiol (1985). [Journal Article; Research Support, N.I.H., Extramural; Research Support, Non-U.S. Gov't]. 2011 2011-12-01;111(6):1644-53.

20. McSharry D, O'Connor C, McNicholas T, Langran S, O'Sullivan M, Lowery M, et al. Genioglossus fatigue in obstructive sleep apnea. Respir Physiol Neurobiol. [Journal Article]. 2012 2012-0815;183(2):59-66.

21. Bradford A, McGuire M, O'Halloran KD. Does episodic hypoxia affect upper airway dilator muscle function? Implications for the pathophysiology of obstructive sleep apnoea. Respir Physiol Neurobiol. [Journal Article; Research Support, Non-U.S. Gov't; Review]. 2005 2005-07-28;147(2-3):22334.

22. Fuller DD, Fregosi RF. Fatiguing contractions of tongue protrudor and retractor muscles: influence of systemic hypoxia. J Appl Physiol (1985). [Journal Article; Research Support, U.S. Gov't, P.H.S.]. 2000 2000-06-01;88(6):2123-30.

23. Pae EK, Wu J, Nguyen D, Monti R, Harper RM. Geniohyoid muscle properties and myosin heavy chain composition are altered after short-term intermittent hypoxic exposure. J Appl Physiol (1985). [Comparative Study; Journal Article; Research Support, N.I.H., Extramural; Research Support, Non-U.S. Gov't; Research Support, U.S. Gov't, P.H.S.]. 2005 2005-03-01;98(3):889-94.

24. Chen LB. Mitochondrial membrane potential in living cells. Annu Rev Cell Biol. [Journal Article; Research Support, U.S. Gov't, P.H.S.; Review]. 1988 1988-01-19;4:155-81.

25. Huttemann M, Helling S, Sanderson TH, Sinkler C, Samavati L, Mahapatra G, et al. Regulation of mitochondrial respiration and apoptosis through cell signaling: cytochrome c oxidase and cytochrome $\mathrm{c}$ in ischemia/reperfusion injury and inflammation. Biochim Biophys Acta. [Journal Article; Research Support, N.I.H., Extramural; Research Support, Non-U.S. Gov't; Review]. 2012 201204-01;1817(4):598-609.

26. Okabe S, Hida W, Kikuchi Y, Kurosawa H, Midorikawa J, Chonan T, et al. Upper airway muscle activity during sustained hypoxia in awake humans. J Appl Physiol (1985). [Journal Article]. 1993 1993-1001;75(4):1552-8.

27. Magalhaes J, Ascensao A, Soares JM, Ferreira R, Neuparth MJ, Marques F, et al. Acute and severe hypobaric hypoxia increases oxidative stress and impairs mitochondrial function in mouse skeletal 
muscle. J Appl Physiol (1985). [Journal Article]. 2005 2005-10-01;99(4):1247-53.

28. Magalhaes J, Ferreira R, Neuparth MJ, Oliveira PJ, Marques F, Ascensao A. Vitamin E prevents hypobaric hypoxia-induced mitochondrial dysfunction in skeletal muscle. Clin Sci (Lond). [Journal Article; Research Support, Non-U.S. Gov't]. 2007 2007-12-01;113(12):459-66.

29. Mezzanotte WS, Tangel DJ, White DP. Waking genioglossal electromyogram in sleep apnea patients versus normal controls (a neuromuscular compensatory mechanism). J CLIN INVEST. [Journal Article; Research Support, U.S. Gov't, Non-P.H.S.; Research Support, U.S. Gov't, P.H.S.]. 1992 1992-0501;89(5):1571-9.

30. Fogel RB, Malhotra A, Pillar G, Edwards JK, Beauregard J, Shea SA, et al. Genioglossal activation in patients with obstructive sleep apnea versus control subjects. Mechanisms of muscle control. Am J Respir Crit Care Med. [Journal Article; Research Support, Non-U.S. Gov't; Research Support, U.S. Gov't, P.H.S.]. 2001 2001-12-01;164(11):2025-30.

31. Fogel RB, Trinder J, White DP, Malhotra A, Raneri J, Schory K, et al. The effect of sleep onset on upper airway muscle activity in patients with sleep apnoea versus controls. J Physiol. [Comparative Study; Journal Article; Research Support, N.I.H., Extramural; Research Support, U.S. Gov't, P.H.S.]. 2005 200504-15;564(Pt 2):549-62.

32. Fogel RB, Malhotra A, Pillar G, Edwards JK, Beauregard J, Shea SA, et al. Genioglossal activation in patients with obstructive sleep apnea versus control subjects. Mechanisms of muscle control. Am J Respir Crit Care Med. [Journal Article; Research Support, Non-U.S. Gov't; Research Support, U.S. Gov't, P.H.S.]. 2001 2001-12-01;164(11):2025-30.

33. Ryan CF, Love LL, Peat D, Fleetham JA, Lowe AA. Mandibular advancement oral appliance therapy for obstructive sleep apnoea: effect on awake calibre of the velopharynx. THORAX. [Journal Article; Research Support, Non-U.S. Gov't]. 1999 1999-11-01;54(11):972-7.

34. Alonso JF, Mananas MA, Rojas M, Bruce EN. Coordination of respiratory muscles assessed by means of nonlinear forecasting of demodulated myographic signals. J Electromyogr Kinesiol. [Journal Article]. 2011 2011-12-01;21(6):1064-73.

35. Jordan AS, Wellman A, Heinzer RC, Lo YL, Schory K, Dover L, et al. Mechanisms used to restore ventilation after partial upper airway collapse during sleep in humans. THORAX. [Journal Article; Research Support, N.I.H., Extramural; Research Support, Non-U.S. Gov't]. 2007 2007-1001;62(10):861-7.

36. Johal A, Gill G, Ferman A, McLaughlin K. The effect of mandibular advancement appliances on awake upper airway and masticatory muscle activity in patients with obstructive sleep apnoea. Clin Physiol Funct Imaging. [Clinical Trial; Journal Article]. 2007 2007-01-01;27(1):47-53.

\section{Figures}




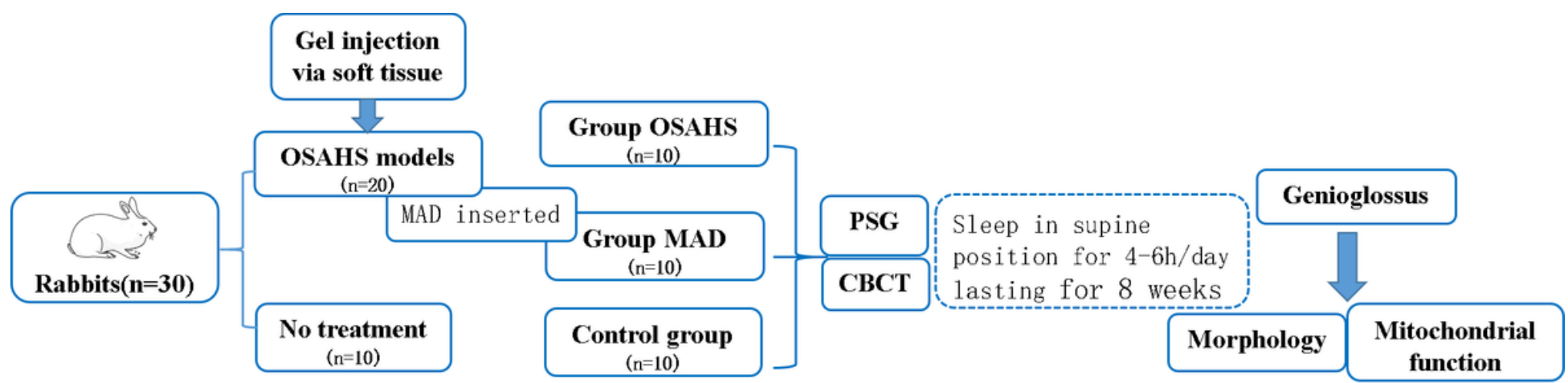

Figure 1

Flowchart of the experimental design. OSAHS was induced by gel injection via the soft palate of New Zealand white rabbits, and then, the rabbits in Group MAD had the MAD inserted. There were three groups: control, OSAHS, and MAD. CBCT scans of the UA and PSG were used to evaluate the effect of MAD treatment. All animals were induced to sleep in a supine position for 4-6 hours per day. After 8 weeks, the genioglossus samples were harvested for evaluation of mitochondrial morphology and function. 

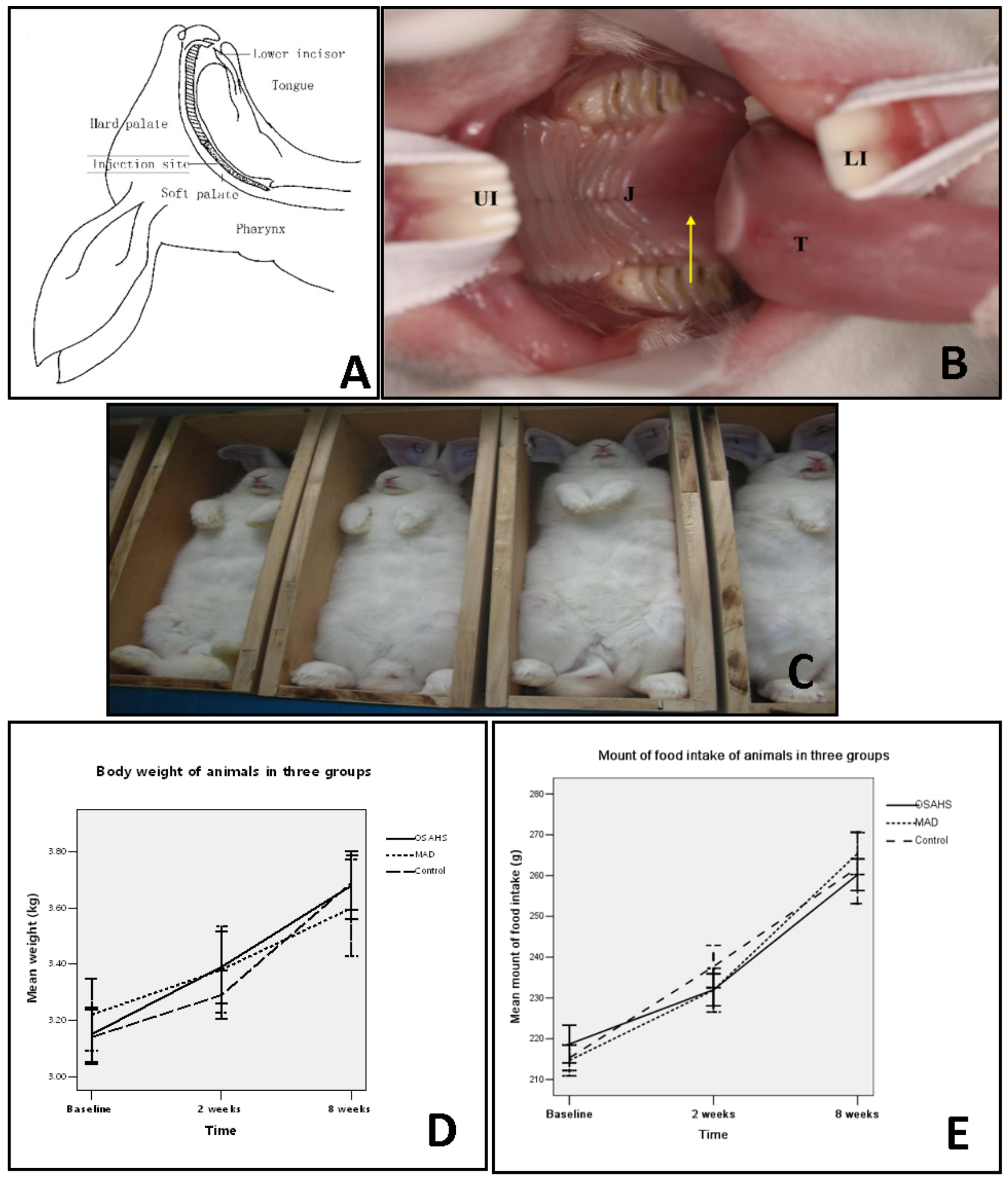

Figure 2

Animal modeling and supine sleep. (A) Diagram of the injection site. (B) The arrow indicates the injection site of the soft palate, approximately $1.5 \mathrm{~cm}$ from the junction of the hard and soft palate. UI means Upper incisors. LI means Lower incisors. J means The junction of the hard and soft palate. T means Tongue. (C) The sleeping rabbits in the supine position were placed in the homemade experimental setup. (D) Body weight of the animals in the three groups. There were no significant differences among the three 
groups. (E) Food intake of the animals in the three groups. There were no significant differences among the three groups.

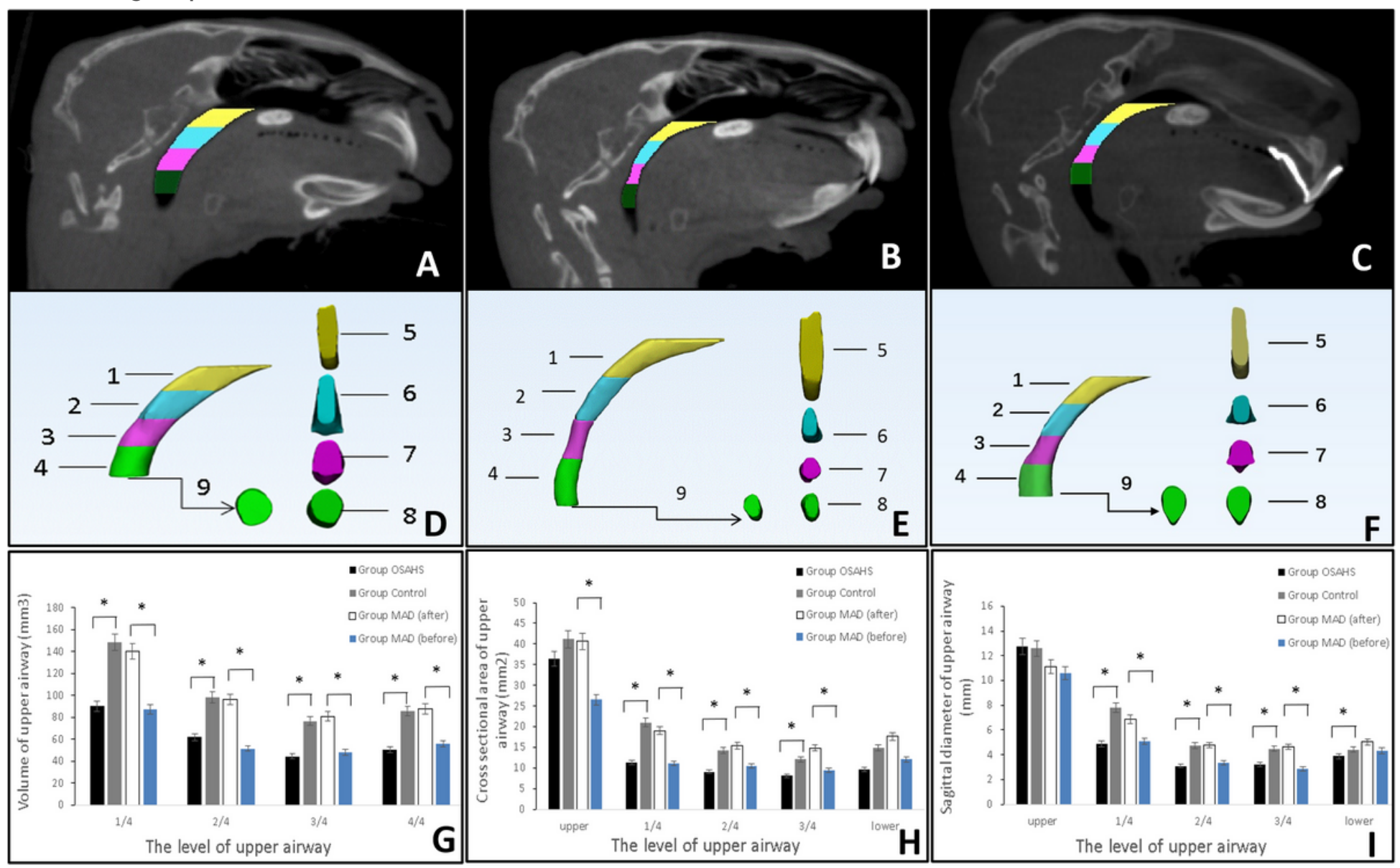

Figure 3

CBCT of the upper airway. Representative 3D images of the UA of the control group (A and D), Group OSAHS ( $\mathrm{B}$ and $\mathrm{E}$ ), and Group MAD ( $\mathrm{C}$ and F). (G) The UA volume significantly decreased in Group OSAHS compared with the control group, and MAD could increase the airway volume. $(\mathrm{H})$ The cross-sectional area significantly decreased in Group OSAHS compared with the control group, and MAD increased the cross-sectional area at the upper, $1 / 4,2 / 4$ and $3 / 4$ levels. (I) The sagittal diameter of the UA significantly decreased in Group OSAHS compared with the control group, and MAD increased the sagittal diameter at the $1 / 4,2 / 4,3 / 4$ and $4 / 4$ levels. ${ }^{*} p<0.05$. 

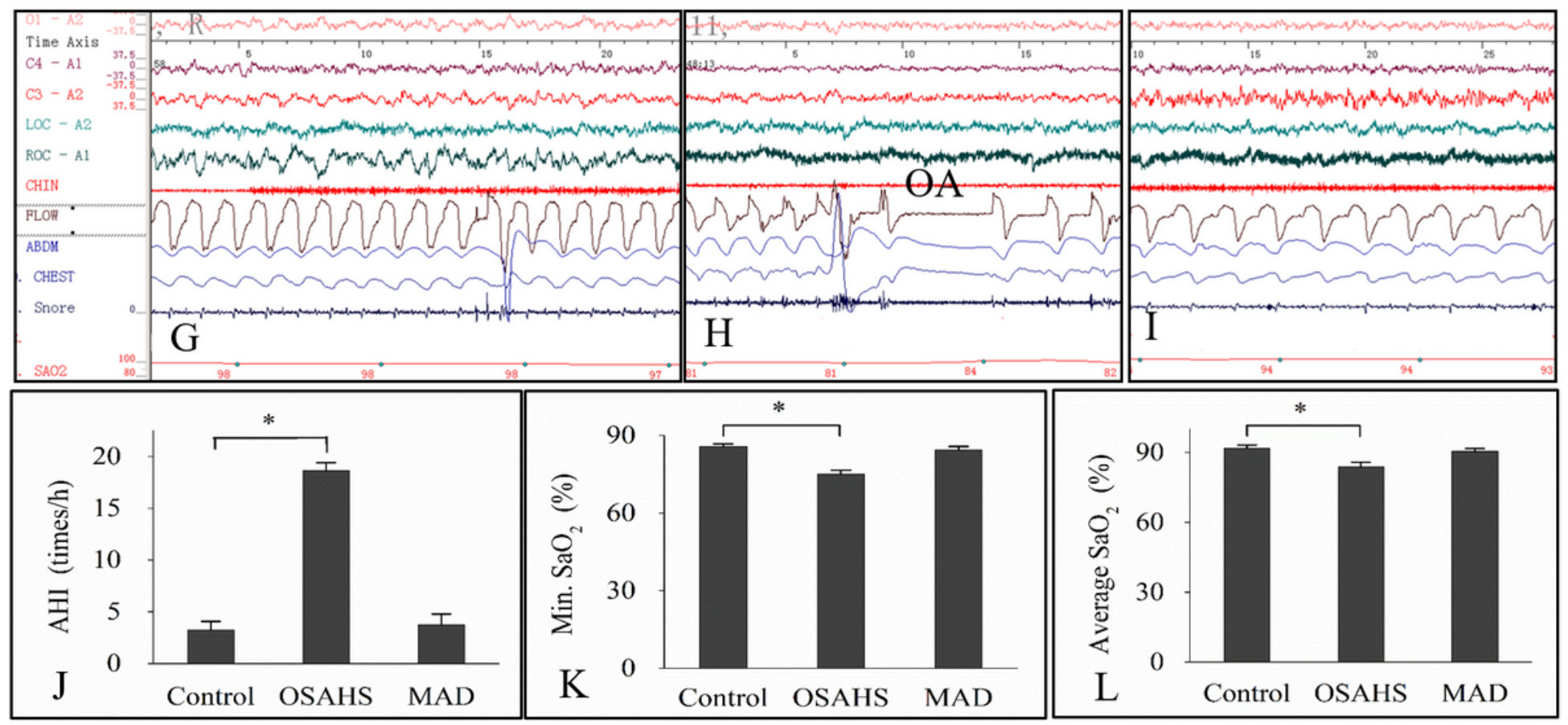

Figure 4

PSG of the three groups. A representative pattern of the $30 \mathrm{~s}$ recordings in the control group (A), Group OSAHS (B), and Group MAD (C). There was a cease in nasal airflow (apnea) with an associated increase in respiratory effort in Group OSAHS (B). The breathing movements and airflow were even and smooth in the control group (A) and Group MAD (C). LOC and ROC: left and right electrooculograms. C3/A2, C4/A1, 01/A2: electroencephalogram leads. FLOW: nasal airflow. CHEST and ABDM: chest and abdominal strain gauges. Snore: microphone recordings of snoring. (C) There was a significant increase in the AHI and a decrease in the minimum $\mathrm{SaO} 2(\mathrm{E})$ and average $\mathrm{SaO} 2(\mathrm{~F})$ in Group OSAHS compared with the control group $(p<0.05)$. The decreased min SaO2 and average SaO2 in Group OSAHS were significantly rescued by MAD treatment ( $E$ and $F$ ). PSG showed that MAD significantly rescued the abnormal respiratory function and sleep parameters. ${ }^{*} \mathrm{p}<0.05$ vs the controls. 


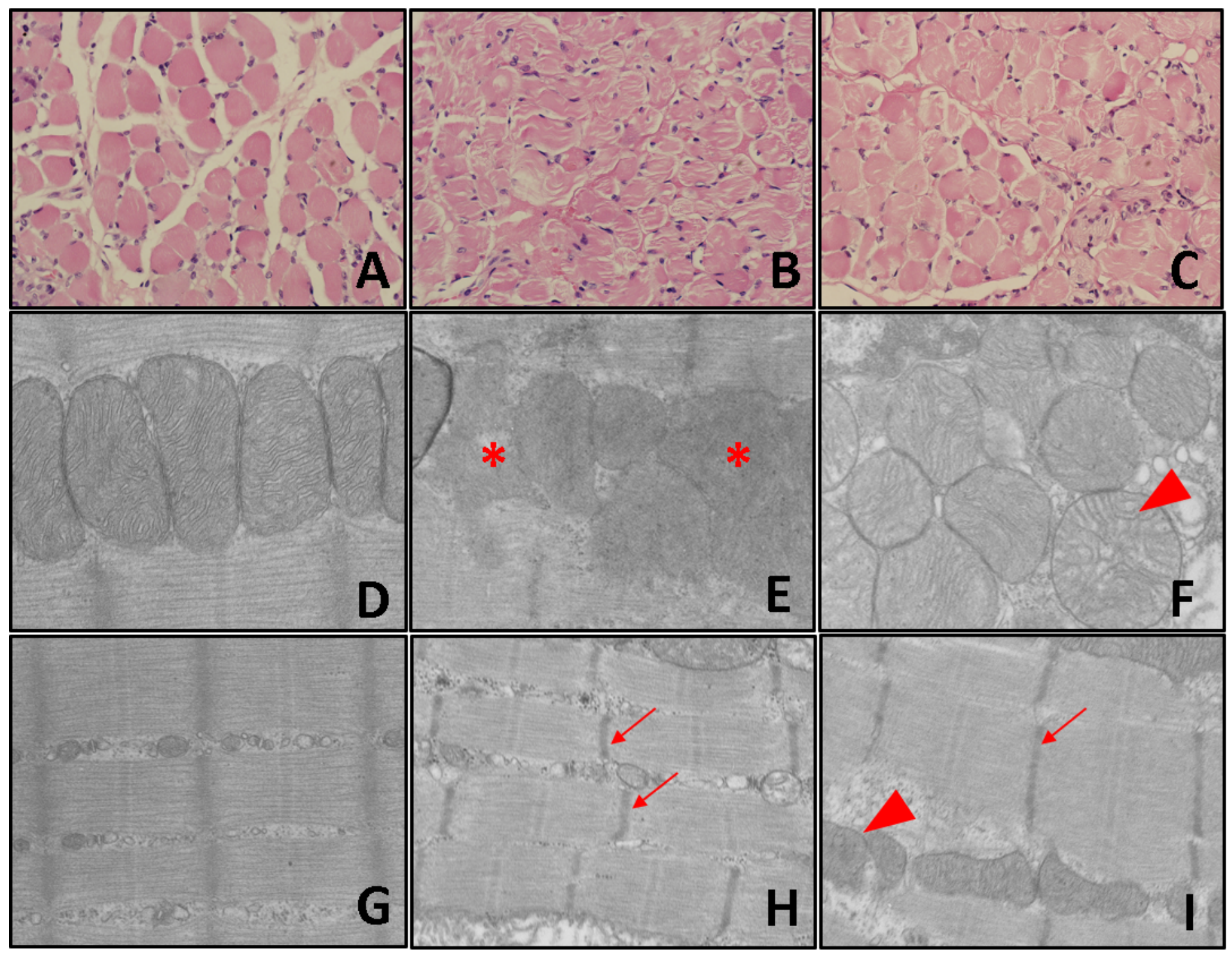

Figure 5

Histopathological changes of the genioglossus in the three groups. Hematoxylin-eosin staining by light microscopy is shown in A-C. (A) The genioglossus fibers were integrated and arranged in an orderly manner in the control group. (B) A disordered arrangement and degeneration of the muscle fibers were observed in Group OSAHS. (C) A mildly disordered arrangement of the genioglossus fibers was detected in Group MAD. The ultrastructure of the genioglossus in transmission electron microscopy is shown in DI. (D) The shape of the genioglossus mitochondria was regular with abundant cristae and intact inner and outer membranes in the control group. (E) There were discontinuous myofibrils, swelling and degeneration of the mitochondria, dilation of the mitochondria and disruption of the cristae in Group OSAHS. Fusion and disappearance of the cristae along with the membrane of mitochondria were shown by *(*in E). (F) The ultrastructural changes of the genioglossus in Group MAD were decreased, with mild mitochondrial degeneration and edema ( red triangle in F ) . (G) There were regular myofibrils, clearly discernible dark bands, $\mathrm{H}$ bands, and $\mathrm{M}$ lines. $(\mathrm{H})$ The disordered structure of the myofibrils in Group OSAHS is shown by arrows ( red arrow in $\mathrm{H}$ ). (I) A mild disordered arrangement of the myofibrils was 
observed in the MAD group( red arrow in I). These results suggested that the genioglossus injury associated with OSAHS can be rescued by MAD treatment.

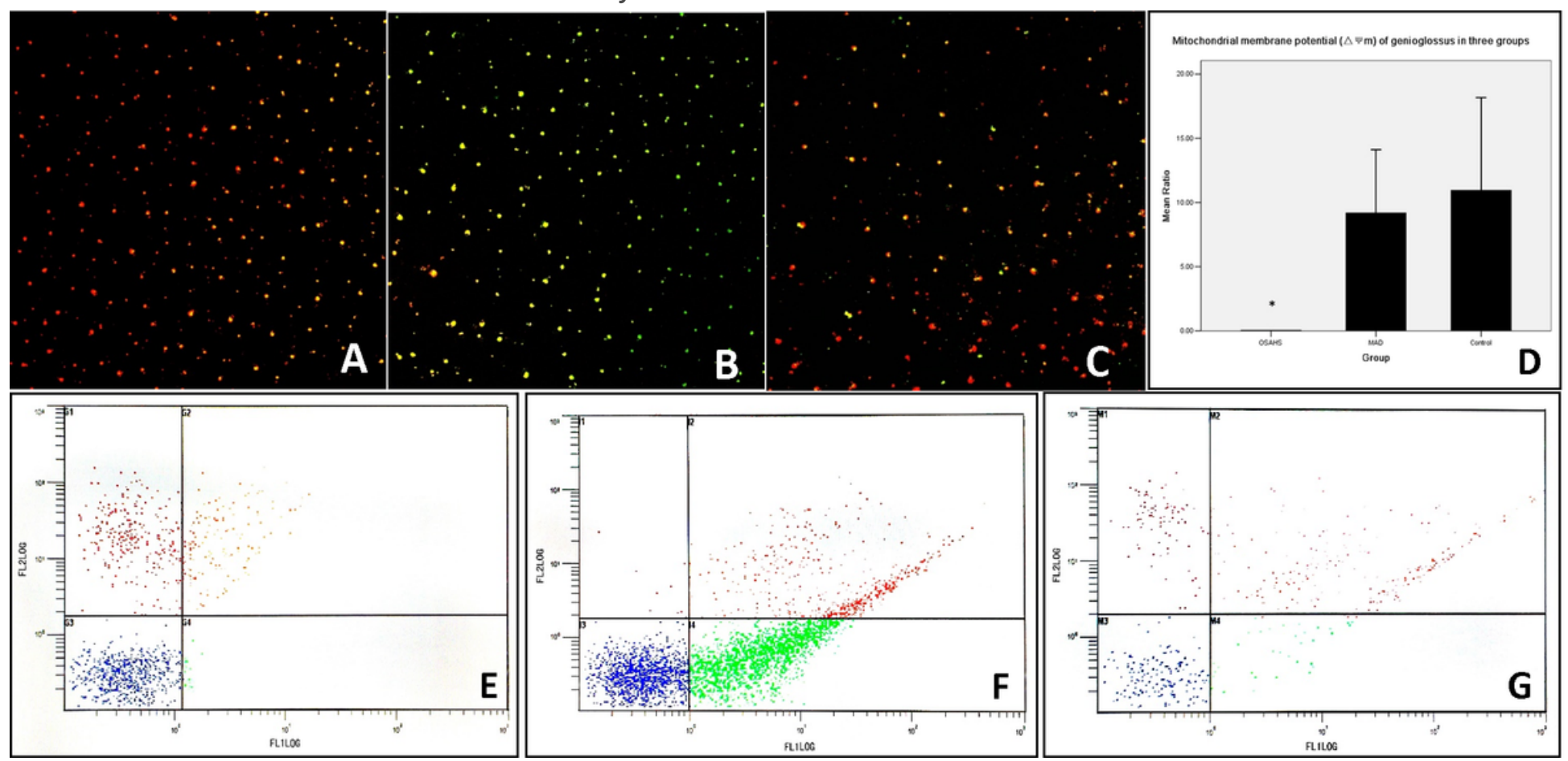

Figure 6

Laser scanning confocal microscopy and flow cytometric analysis. (A) The mitochondrial membrane potential $(\triangle \Psi \mathrm{m})$ of the genioglossus was high in the control group, with mostly red fluorescence. (B) $\triangle \Psi_{\mathrm{m}}$ of the genioglossus was decreased in Group OSAHS, with green fluorescence. (C) $\triangle \Psi_{\mathrm{m}}$ of the genioglossus was high in Group MAD, and green fluorescence was rare, with mostly red fluorescence. The flow diagram shows that cells labeled with green fluorescence could be observed in Q3 of the graphics, and cells labeled with red fluorescence could be observed in Q1 of the graphics. (D) $\triangle \Psi \mathrm{m}$ of the genioglossus was significantly lower in Group OSAHS than in the control group, and there was no significant difference between the control group and Group MAD. The asterisk * represents $p<0.05$ compared with the controls. $(E)$ There was a high percentage of cells labeled with red fluorescence in the control group. (F) There was a high percentage of cells labeled with green fluorescence in Group OSAHS. (G) There was a high percentage of cells labeled with red fluorescence in Group MAD. 


\section{Complex activity in three groups}

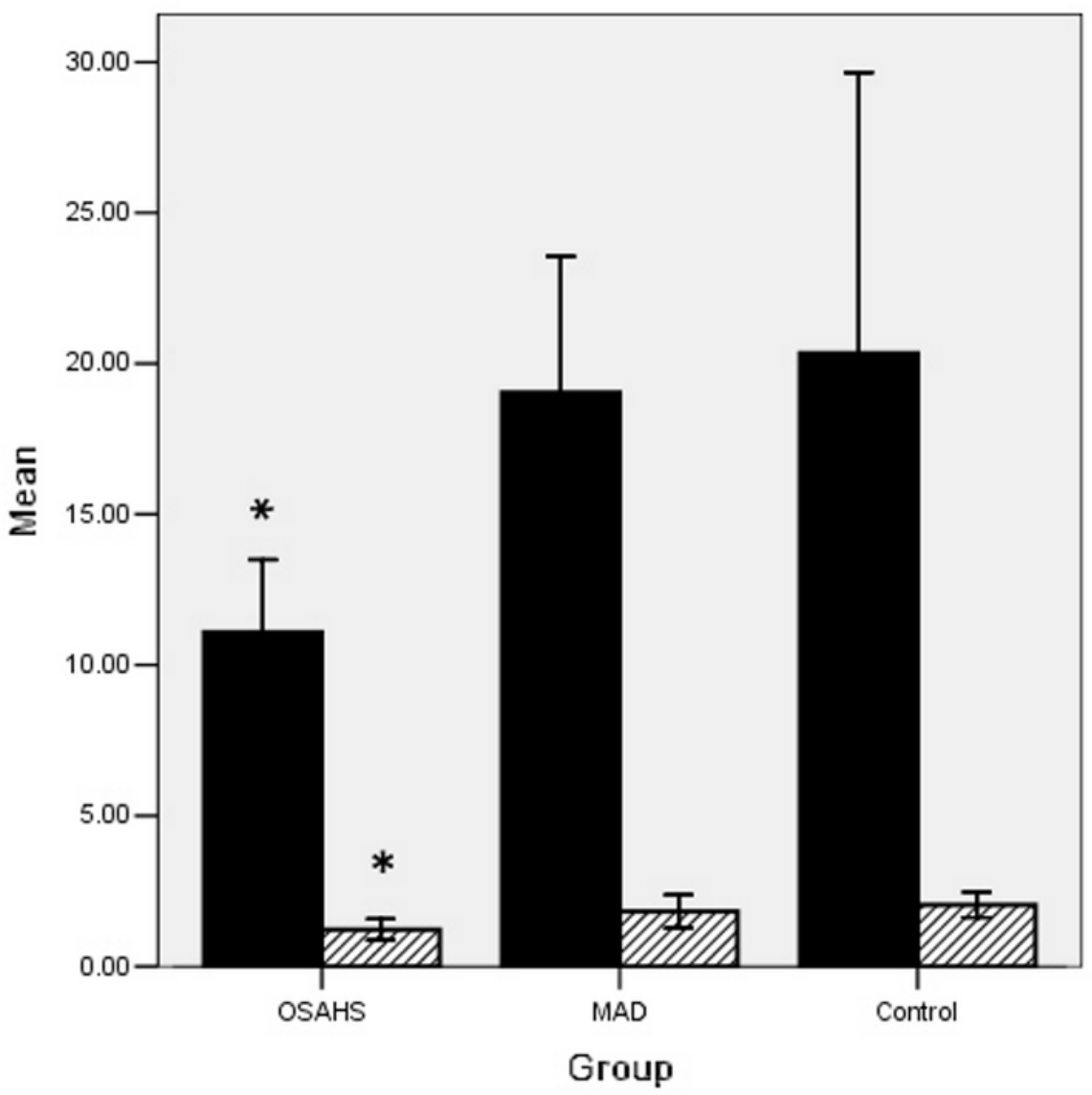

ComplexI (micromoles NADH/min) 口 Complex IV (micromolar cytochrome $\mathrm{C} / \mathrm{min}$ )

\section{Figure 7}

Complex I and complex IV activities of the genioglossus in the three groups. Complex I and complex IV activities of the genioglossus were significantly lower in Group OSAHS than in the control group, and there was no significant difference between the control group and Group MAD. The asterisk * represents $p<0.05$ compared with the controls, the bars in the figures are SDs

\section{Supplementary Files}

This is a list of supplementary files associated with this preprint. Click to download.

- Additionalfile1.doc

- Additionalfile2.doc 